\section{Isolation of Plasma Membranes from the Nervous System by Countercurrent Distribution in Aqueous Polymer Two-Phase Systems}

\author{
Jens Schindler and Hans Gerd Nothwang
}

\section{Summary}

The plasma membrane separates the cell-interior from the cell's environment. To maintain homeostatic conditions and to enable transfer of information, the plasma membrane is equipped with a variety of different proteins such as transporters, channels, and receptors. The kind and number of plasma membrane proteins are a characteristic of each cell type. Owing to their location, plasma membrane proteins also represent a plethora of drug targets. Their importance has entailed many studies aiming at their proteomic identification and characterization. Therefore, protocols are required that enable their purification in high purity and quantity. Here, we report a protocol, based on aqueous polymer two-phase systems, which fulfils these demands. Furthermore, the protocol is time-saving and protects protein structure and function.

Key words: Brain, Plasma membrane, Countercurrent distribution, Two-phase system, Enrichment

2

1. Introduction

Plasma membrane (PM) proteins mediate signal transduction, solute transport, secretion, and cell-cell contact. They are also the central players in the propagation and transmission of action potentials, which are the lingua franca in the nervous system. Finally, $\sim 70 \%$ of all known drug targets act on them (1). PM proteins are thus of prime interest in many areas of both basic and biomedical research. However, their proteome analysis is rather difficult, as they encompass only $0.4-2.5 \%$ of the total cellular protein amount (2). This renders their identification difficult in the bulk of other, more abundant proteins of the cytoskeleton, 
the energy metabolism, and alike. Furthermore, many properties of PMs such as density overlap with those of other membranous compartments, mainly the endoplasmic reticulum. Classical purification protocols of PM proteins are therefore rather cumbersome and material-consuming. This is for instance the case with the most popular method, consisting of a combination of differential and density gradient centrifugation steps $(3,4)$. Other methods are either quite expensive and contamination-prone (e.g. immunoprecipitation) or apply only to cultured cells such as surface labeling, which cannot be applied to bulky tissue.

Interest in novel, more efficient subcellular purification protocols has recently emerged from the impressive progress in the analytical part of proteomics. Current mass spectrometry can identify proteins at concentrations of less than $1 \mathrm{pM}$. This allows for detection of proteins such as the neuronal PSD-95, which is present at 300 copies/postsynaptic density (Chen et al., AU1 2005 ) in as few as $2 \times 10^{6}$ neurons, based on 1,000 postsynaptic densities/cells. Hence, proteomics studies on functional or anatomically well-characterized small tissue samples or scarce biopsy material come into reach. However, protocols for the isolation of defined subcellular compartments did not keep pace, despite the recognized need to analyze the subproteome of the various compartments separately. This cellular dissection is mandatory to detect low abundant proteins and to identify compartment-specific post-translational modifications or significant changes in protein localization. Changes therein often underlie physiological and pathophysiological processes (5).

A highly selective and efficient method to separate membranes of different subcellular origin was developed more than 30 years ago by Albertsson and colleagues and was based on the use of aqueous polymer two-phase systems $(6,7)$. These systems often form, when aqueous solutions of two structural different water-soluble polymers are mixed above a defined concentration. Most often, poly(ethylene glycol) (PEG) and dextran are used as polymers, as they are cheap, require only moderate concentrations, separate easily, and preserve protein structure and function well. When mixed, phases will form, and the upper phase will be enriched in PEG, whereas the bottom phase will mainly contain dextran. Interestingly, the various cellular membranes have different affinities to partition in either of the two phases. PMs prefer the upper phase, whereas mitochondria partition rather to the bottom phase. This different behavior can be attributed to differences in hydrophobic and hydrophilic surface properties of membranes, most likely arising from differences in their phosholipid composition.

Membranes differ only subtly in their surface properties and the isolation of PMs by aqueous polymer two-phase systems cannot be achieved in a single step. One possibility to increase the 
Isolation of Plasma Membranes from the Nervous System by Countercurrent

purity of the PM fraction is multistep extraction procedures such as countercurrent distribution (CD) (Fig. 1). CD is based on the Nernst distribution law. Membranes are separated by this method on the basis of their different solubilities in two immiscible aqueous solutions of structurally different polymers. These two phases, flowing into opposite directions, are brought into contact, mixed, and allowed to separate. PMs preferentially partition to the top phase and will reside there throughout the multiple extractions. Intracellular membranes such as mitochondria will preferentially partition to the bottom phase throughout the procedure. We recently adapted this principle to the isolation of

76 77 78 79 80 81 82 83 84 85 86

I.

$$
\text { two-phase system }
$$
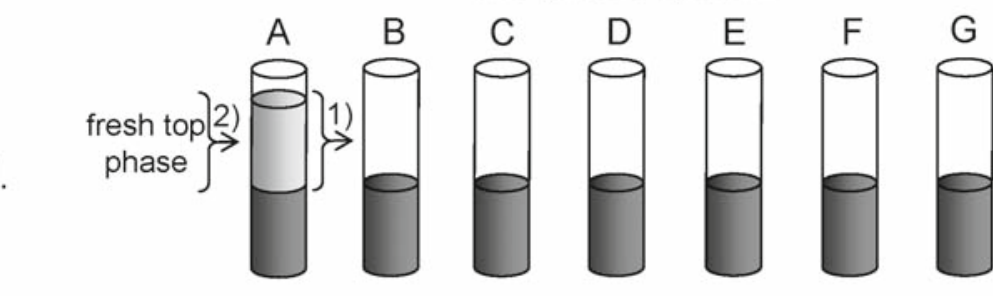

II.
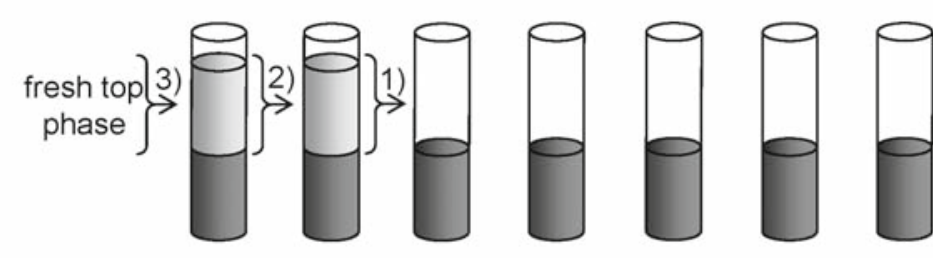

III.
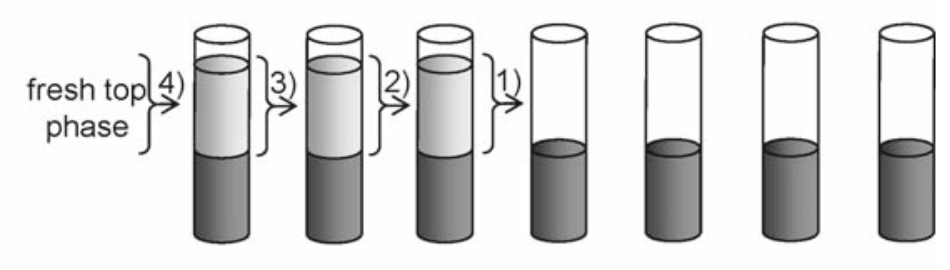

$$
\vdots
$$

VII.

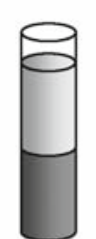

A

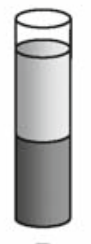

$\mathrm{B}$

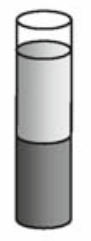

C

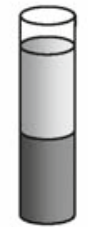

D

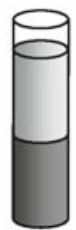

E

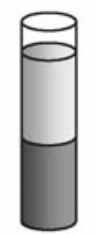

F

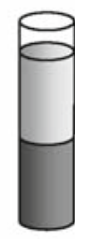

G

Fig. 1. Scheme of countercurrent distribution. In CD experiments, the top-phase of the first two-phase system $A$ is transferred to a fresh bottom-phase $B$ and the bottomphase of two-phase system $A$ is re-extracted with a fresh top-phase. After six iterations, biomaterial is efficiently separated. 
Schindler and Nothwang

87

88

89

90

91

92

93

94

95

96

97

98
PMs from the nervous system (8). The protocol is fast, easy to perform, and yields up to $30 \%$ of the initial PMs with high purity. Contaminations by endoplasmic reticulum and mitochondria, the major contaminations in standard protocols, were low.

\section{Materials}

\subsection{Two-Phase Systems}

Owing to the strong influence of ions on membrane partitioning in the two-phase systems, double distilled water should be used throughout the experiments.

1. Glass-Teflon homogenizer.

2. Dextran stock solution: Dextran T500 (20\%,w/w) ( see Note $\mathbf{1})$.

3. PEG stock solution: PEG $3350(40 \%, w / w)$.

4. Tris- $\mathrm{H}_{2} \mathrm{SO}_{4}$ : Tris $(200 \mathrm{mM}), \mathrm{pH} 7.8$ adjusted with $\mathrm{H}_{2} \mathrm{SO}_{4}$.

\section{Methods}

3.1. Two-Phase Partitioning
All steps of the affinity two-phase partitioning protocol should be performed at $4^{\circ} \mathrm{C}$. Working at room temperature prevents phase separation. The procedure is illustrated in Fig. 1. The numbers in Fig. 1 correspond to the numbered two-phase systems given in Table $\mathbf{1}$, and the letters refer to the phases as indicated in the protocol given below.

1. Prepare seven two-phase systems with the compositions indicated in Table 1, 1 day prior to use. Mix them by 20 invertations, vortexing for $10 \mathrm{~s}$, another 20 invertations, and store

\section{Table 1}

\section{Composition of two-phase systems}

\begin{tabular}{lll} 
& Two-phase system “A” (g) & Two-phase systems “B-G” (g) \\
\hline Dextran stock solution & 1.035 & 1.035 \\
PEG stock solution & 0.518 & 0.518 \\
Tris- $\mathrm{H}_{2} \mathrm{SO}_{4}$ & 0.750 & 0.750 \\
Water & 0.598 & 0.698 \\
\hline
\end{tabular}


Isolation of Plasma Membranes from the Nervous System by Countercurrent

the mixtures at $4^{\circ} \mathrm{C}$ overnight. Two-phase gystems will form overnight with the top phase enriched in PEG and the bottom phase enriched in dextran.

109

110

2. On the next day, remove all top phases from two-phase systems "B-G" and store them separately.

3. Homogenize $0.1 \mathrm{~g}$ brain tissue in two-phase system " $\mathrm{A}$ " using a glass-Teflon homogenizer followed by $45 \mathrm{~s}$ of sonication. Centrifuge at $700 \times g$ for $5 \mathrm{~min}$ to accelerate phase separation.

114

115

4. Transfer the top phase (Top) of two-phase system "A" onto the bottom-phase (Bot) of two-phase system "B" (TopA $\rightarrow$ BotB) (see Note 2). Add an equal amount of fresh top-phase (stored in step 2) onto bottom-phase "A" (I. in Fig. 1). Mix both two-phase systems by 20 invertations, vortex for $10 \mathrm{~s}$, then mix again by another 20 invertations. Centrifuge at $700 \times \mathfrak{g}$ for 5 min to accelerate phase separation.

5. Transfer top phases in the following order (II. in Fig. 1): (1) 124 TopB $\rightarrow$ BotC; (2) TopA $\rightarrow$ BotB. Add an equal amount of 125 fresh top-phase (stored in step 2) onto bottom-phase "A." 126 Mix all two-phase systems by 20 invertations, vortexing for $10 \quad 127$ $\mathrm{s}$, and another 20 invertations. Centrifuge at $700 \times g$ for $5 \mathrm{~min} \quad 128$ to accelerate phase separation.

6. Transfer top phases in the following order (III. in Fig. 1): 130 (1) TopC $\rightarrow$ BotD; (2) TopB $\rightarrow$ BotC; (3) Top A $\rightarrow$ BotB. Add 131 an equal amount of fresh top-phase (stored in step 2) onto 132 bottom-phase "A."

Mix all two-phase systems by 20 invertations, vortexing for $10 \mathrm{~s}$, and another 20 invertations. Centrifuge at $700 \times \mathfrak{g}$ for 5 min to accelerate phase separation.

7. Transfer top phases in the following order: (1) Top D $\rightarrow$ BotE; (2) TopC $\rightarrow$ BotD; (3) TopB $\rightarrow$ BotC; (4) Top A $\rightarrow$ BotB. Add an equal amount of fresh top-phase (stored in step 2) onto bottom-phase "A." Mix all two-phase systems by 20 invertations, vortexing for $10 \mathrm{~s}$, and another 20 invertations. Centrifuge at $700 \times g$ for $5 \mathrm{~min}$ to accelerate phase separation.

133

134 135 136

8. Transfer top phases in the following order: (1) TopE $\rightarrow$ BotF; (2) Top D $\rightarrow$ BotE; (3) TopC $\rightarrow$ BotD; (4) TopB $\rightarrow$ BotC; (5) Top $\mathrm{A} \rightarrow$ BotB. Add an equal amount of fresh top-phase (stored in step 2) onto bottom-phase "A." Mix all two-phase systems by 20 invertations, vortexing for $10 \mathrm{~s}$, and another 20 invertations. Centrifuge at $700 \times g$ for $5 \mathrm{~min}$ to accelerate phase separation.

9. Transfer top phases in the following order: (1) TopF $\rightarrow$ BotG: (2) TopE $\rightarrow$ BotF; (3) Top D $\rightarrow$ BotE; (4) TopC $\rightarrow$ BotD; (5) TopB $\rightarrow$ BotC; (6) Top A $\rightarrow$ BotB. Add an equal amount of fresh top-phase (stored in step 2 ) onto bottom-phase "A." $10 \mathrm{~s}$, and another 20 invertations. Centrifuge at $700 \times$ gfor 5 
Schindler and Nothwang

\section{Notes}

min to accelerate phase separation. After phase separation, you end up with seven two-phase systems (VII. in Fig. 1). PMs are enriched in TopF and TopG.

10. PMs can be recovered from TopG or combined TopF $+\mathrm{G}$ (see Note 3) by diluting the phases 1:10 with water followed by ultracentrifugation at $1,50,000 \times g$ and $4^{\circ} \mathrm{C}$ for $1 \mathrm{~h}$.

\section{Acknowledgements}

1. Dextran can contain up to $10 \%$ water and for that reason has to be freeze-dried. For freeze-drying, dissolve dextran in distilled water in a plastic dish with a large surface (e.g. Petri dish), freeze it at $-80^{\circ} \mathrm{C}$, and dry it by sublimating the water under vacuum. Store the freeze-dried dextran in closed plastic tubes sealed tightly with parafilm at $-20^{\circ} \mathrm{C}$. Let it come to room temperature before opening to protect it from humidity.

2. In two-phase systems, interphases are always considered as part of the bottom phase.

3. The purity of PMs from TopG alone is slightly higher but combining TopF and TopG nearly doubles the yield.

This work was funded in part by the Nano+Bio-Center of the University of Kaiserslautern.

\section{References}

1. Hopkins AL, Groom CR. The druggable genome. Nat Rev Drug Discov 2002 Sep; 1:727-30.

2. Evans WH. Isolation and characterization of membranes and cell organelles. In: Rickwood D, editor. Preparative Centrifugation. lst ed. Oxford: IRL Press; 1991. p. 233-70.

3. DePierre JW, Karnovsky ML. Isolation of a nuclear fraction from guinea pig polymorphonuclear leukocytes after controlled hypotonic homogenization. Biochim Biophys Acta 1973 Aug 17; 320(1):205-9.

4. Cao R, Li X, Liu Z, Peng X, Hu W, Wang X, et al. Integration of a two-phase partition method into proteomics research on rat liver plasma membrane proteins. J Proteome Res 2006 Mar; 5:634-42.

5. Dreger M. Subcellular proteomics. Mass Spectrom Rev 2003 Jan; 22(1):27-56.

6. Albertsson PA. Partition of Cell Particles and Macromolecules. 3rd ed. New York: Wiley; 1986.

7. Schindler J, Nothwang HG. Aqueous polymer twophase systems: Effective tools for plasma membrane proteomics. Proteomics 2006 Oct; 6:5409-17.

8. Schindler J, Lewandrowski U, Sickmann A, [Au2] Friauf E. Aqueous polymer two-phase systems for the proteomic analysis of plasma membranes from minute brain samples $\wedge$ I Proteane Res 2007;in press. 


\section{Author Queries}

Chapter No.:19

0000951469

\begin{tabular}{|l|l|l|}
\hline Queies & Details Required & Author's Response \\
\hline AU1 & $\begin{array}{l}\text { Please update with complete details and move the ref to the reference list and } \\
\text { use the number only here. }\end{array}$ & \\
\hline AU2 & Please update. & \\
\hline
\end{tabular}

